\title{
The Bernoulli Equation and the Poloidal-sonic Singularity in an Inverse Aspect-ratio Expansion Formulation of Equilibria with Flow ${ }^{*}$
}

\author{
Daniel RABURN and Atsushi FUKUYAMA \\ Kyoto University, Kyoto 606-8501, Japan
}

(Received 6 December 2010 / Accepted 10 February 2011)

\begin{abstract}
Fluid flow can play a significant role in plasma equilibrium, possibly producing transport barriers and profile pedestals. To be consistent with hot ions, finite Larmor radius (FLR) effects should be included. Ito and Nakajima have developed a formulation for calculating magnetohydrodynamic (MHD) equilibria with poloidal-sonic flow and FLR effects using an inverse aspect-ratio expansion, known as the "Ito formulation" [A. Ito and N. Nakajima, AIP Conference Proceedings 1069, 121 (2008)]. The Ito formulation typically possess a singularity when the poloidal flow varies from sub- to super-poloidal sonic, known as the "poloidal-sonic (PS) singularity." The presence of the PS singularity prevents the Ito formulation from being directly applied to equilibria with such flows. An investigation of the single-fluid MHD model shows that the PS singularity is due to an inverse aspectratio expansion of the Bernoulli equation. This suggests that, in order to use the Ito formulation for an accurate calculation of MHD equilibria with poloidal-sonic flows, the Bernoulli equation must be handled non-linearly near the singularity.
\end{abstract}

(C) 2011 The Japan Society of Plasma Science and Nuclear Fusion Research

Keywords: magnetohydrodynamics, equilibrium, flow, poloidal-sonic singularity, Bernoulli equation, two-fluid model, finite Larmor radius

DOI: $10.1585 /$ pfr.6.2403044

\section{Introduction}

Equilibrium flow can be important for macroscopic stability and energy confinement [1]. For single-fluid magnetohydrodynamics (MHD), equilibria with flow can be calculated by solving a generalized Grad-Shafranov (GS) and Bernoulli equation [2,3]. Under the single-fluid MHD model, poloidal flows which vary from sub- to superpoloidal-sonic can produce profile pedestals $[2,4]$. We refer to such flows as "poloidal-transonic" and the pedestal as the "poloidal-sonic (PS) discontinuity." However, such calculations are not consistent with hot ions, which produce small-scale length effects not included in the singlefluid MHD model.

Ito and Nakajima have developed a formulation for MHD equilibria with flow which includes ion finite Larmor radius (FLR) and two-fluid Hall MHD effects [5]. We refer to this as the "Ito formulation." The formulation uses an expansion in the inverse aspect-ratio $(\varepsilon)$ and treats flows on the order of the poloidal sound speed, and equilibrium is governed by equations for the first- and second-order poloidal flux. The formulation typically possesses a singularity for poloidal-transonic flows. We refer to this as the "poloidal-sonic (PS) singularity." An overview of the Ito formulation is presented in Sec. 2. Further information on the Ito formulation, included an analysis of the criteria

author's e-mail: draburn@p-grp.nucleng.kyoto-u.ac.jp

*) This article is based on the presentation at the 20th International Toki Conference (ITC20). governing the presence of the PS singularity, is presented in Ref. [6].

The presence of the PS singularity prevents the Ito formulation from being applied directly to the calculation of equilibria with poloidal-transonic flows $[7,8]$. In order to better understand the PS singularity in the Ito formulation, we have compared the Ito formulation to a formulation for single-fluid MHD equilibria without using an expansion in $\varepsilon$. We have derived a set of equations governing such equilibria. These equations describe the same physics as those derived by other authors, such as Hameiri [9]. However, the formulations in the existing literature include a large number of abstract quantities; in our formulation, we instead use quantities with simpler physical meanings. The formulation and an overview of the derivation is presented in Sec. 3.

The formulation derived in Sec. 3 includes the Bernoulli equation, which governs the relationship between the flow and density. For a given flow, the Bernoulli equation can possess zero, one, or two real roots for the density. If there are no real roots, then the given flow is physically impossible. The single root occurs at the "poloidal-transonic surface," where the poloidal flow changes from sub- to super-poloidal-sonic [3]. In Sec.4, we examine the Bernoulli equation under a certain subset of the ordering imposed by the Ito formulation, and we show that the PS singularity is reproduced. 
In Sec. 5, we state our conclusions regarding how the Ito formulation would need to be modified in order to be applied to the calculation of MHD equilibria with poloidaltransonic flows, FLR effects, and two-fluid Hall MHD effects.

\section{The Ito Formulation}

Two-fluid MHD equilibria with flow and hot ions are described by the following set of equations [5]:

$$
\begin{aligned}
& \boldsymbol{\nabla} \cdot \boldsymbol{B}=0, \\
& \boldsymbol{\nabla} \times \boldsymbol{B}=\mu_{0} \boldsymbol{j}, \\
& \boldsymbol{\nabla} \times \boldsymbol{E}=0, \\
& \boldsymbol{\nabla} \cdot(n \boldsymbol{v})=0, \\
& \boldsymbol{E}+\boldsymbol{v} \times \boldsymbol{B}=\lambda_{\mathrm{H}}\left(\boldsymbol{j} \times \boldsymbol{B}-\boldsymbol{\nabla} p_{\mathrm{e}}\right) /(e n), \\
& \boldsymbol{v}_{\mathrm{e}} \equiv \boldsymbol{v}-\lambda_{\mathrm{H}} \boldsymbol{j} /(e n), \\
& \boldsymbol{v}_{\mathrm{e}} \cdot \boldsymbol{\nabla} p_{\mathrm{e}}+\gamma p_{\mathrm{e}} \boldsymbol{\nabla} \cdot \boldsymbol{v}_{\mathrm{e}}=-\frac{2}{5} \lambda_{\mathrm{i}} \gamma \boldsymbol{\nabla} \cdot \boldsymbol{q}_{\mathrm{e}}, \\
& \boldsymbol{v} \cdot \boldsymbol{\nabla} p_{\mathrm{i}}+\gamma p_{\mathrm{i}} \boldsymbol{\nabla} \cdot \boldsymbol{v}=-\frac{2}{5} \lambda_{\mathrm{e}} \gamma \boldsymbol{\nabla} \cdot \boldsymbol{q}_{\mathrm{i}}, \\
& \boldsymbol{j} \times \boldsymbol{B}-\boldsymbol{\nabla}\left(p_{\mathrm{i}}+p_{\mathrm{e}}\right)=m_{\mathrm{i}} n \boldsymbol{v} \cdot \boldsymbol{\nabla} \boldsymbol{v}+\lambda_{\mathrm{i}} \boldsymbol{\nabla} \cdot \boldsymbol{\Pi}_{\mathrm{i}}^{\mathrm{gv}},
\end{aligned}
$$

where $\boldsymbol{B}$ is the magnetic field, $\mu_{0}$ is the permeability of free space, $\boldsymbol{j}$ is the electric current density, $\boldsymbol{E}$ is the electric field, $\boldsymbol{v}$ is the (single-fluid) velocity, $p_{\mathrm{e}}$ is the electron pressure, $e$ is the elementary charge, $n$ is the electron density, $m_{\mathrm{i}}$ is the ion mass, $\gamma=5 / 3, q_{\mathrm{e}}$ is the electron heat flux, $p_{\mathrm{i}}$ is the ion pressure, $\boldsymbol{q}_{\mathrm{i}}$ is the ion heat flux, and $\boldsymbol{\Pi}_{\mathrm{i}}^{\mathrm{gv}}$ is the ion gyroviscous tensor. $\lambda_{\mathrm{H}}, \lambda_{\mathrm{i}}$, and $\lambda_{\mathrm{e}}$ are artificial indices which have been introduced to control the two-fluid, nonideal terms.

The Ito formulation is relevant for high-beta $\left(p_{\mathrm{i}} \sim\right.$ $\left.p_{\mathrm{e}} \sim \varepsilon B^{2} / \mu_{0}\right)$ large aspect-ratio $\left(\varepsilon \equiv a / R_{0} \ll 1\right)$ axisymmetric $(\partial / \partial \phi=0)$ tokamak $\left(B_{\mathrm{pol}} \sim \varepsilon B\right)$ equilibria with flows on the order of the poloidal sound speed $\left(v_{\text {tor }} \lesssim v_{\text {pol }} \sim C_{\mathrm{s}, P}\right)$ :

$$
C_{\mathrm{s}, P} \equiv\left(B_{\mathrm{pol}} / B\right) \sqrt{\gamma T / m_{\mathrm{i}}}
$$

Finally, assuming the slow dynamics ordering [10] implies $\varepsilon \sim \rho_{\mathrm{i}} / a$, where $\rho_{\mathrm{i}}$ is the ion gyroradius.

Equilibrium is governed by five free profiles of the first-order poloidal flux and GS equations for the first- and second-order poloidal flux [6]. Because of the flow, the pressure contours can deviate from the flux contours. The term which governs the deviation can be singular. Under the single-fluid MHD model, the singularity happens when $v_{\text {pol }}=C_{\mathrm{s}, P}$ and is the aforementioned PS singularity.

\section{Axisymmetric Single-Fluid MHD Equilibria with Flow}

In order to better understand the PS singularity in the Ito formulation, we consider a formulation for single-fluid MHD equilibria without using an expansion in $\varepsilon$. In this section, we derive the equilibrium equations for this formulation. In Sec.4, we will compare this formulations with the Ito formulation.
Consider the single-fluid MHD model $\left[\left(\lambda_{\mathrm{H}}, \lambda_{\mathrm{e}}, \lambda_{\mathrm{i}}\right)=\right.$ $(0,0,0)]$. Assume $1<\gamma<\infty$ and use an $(R, \phi, Z)$ coordinate system, where $R$ is the major radius, $\phi$ is the toroidal angle, and $Z$ is the distance above the midplane, with $\hat{\boldsymbol{R}} \times \hat{\boldsymbol{\phi}}=\hat{\boldsymbol{Z}}$. Eqs. (1)-(4) imply:

$$
\begin{aligned}
& \boldsymbol{B}=\boldsymbol{\nabla} \psi \times \nabla \phi+I \nabla \phi, \\
& \mu_{0} \boldsymbol{j}=\boldsymbol{\nabla} I \times \nabla \phi-\Delta^{*} \psi \nabla \phi, \\
& \boldsymbol{E}=-\boldsymbol{\nabla} \Phi, \\
& \boldsymbol{v}=n^{-1} \nabla \Psi \times \nabla \phi+v_{\mathrm{tor}} R \nabla \phi,
\end{aligned}
$$

where $\psi$ is the poloidal flux, $I$ is the poloidal current, $\Phi$ is the scalar potential, $\Psi$ is the stream function, $v_{\text {tor }}$ is the toroidal fluid velocity, and:

$$
\Delta^{*} \equiv \partial^{2} / \partial R^{2}-(1 / R) \partial / \partial R+\partial^{2} / \partial Z^{2}
$$

Define the Poisson bracket $[\ldots . . . . . \rrbracket$ and the flux derivative:

$$
\begin{aligned}
& \llbracket f \mid g \rrbracket \equiv \boldsymbol{\nabla} \phi \cdot(\boldsymbol{\nabla} f \times \boldsymbol{\nabla} g), \\
& f^{\prime} \equiv \boldsymbol{\nabla} \psi \cdot \boldsymbol{\nabla} f /\|\boldsymbol{\nabla} \psi\|^{2},
\end{aligned}
$$

where $f$ and $g$ represent arbitrary scalar functions of $(R, Z)$. Observe:

$$
\llbracket f \mid \psi \rrbracket=0 \quad \Longleftrightarrow \quad f=f(\psi) .
$$

Further, define the "aspect-ratio vector function" $\boldsymbol{\varepsilon}_{\mathrm{F}}$, the single-fluid temperature $T$, and the scalar function $\tau$ :

$$
\begin{aligned}
& \boldsymbol{\varepsilon}_{\mathrm{F}} \equiv \nabla \psi \times \nabla \phi / B=\boldsymbol{B}_{\mathrm{pol}} / B \\
& T \equiv p / n \\
& \tau \equiv p n^{-\gamma}=T n^{1-\gamma}
\end{aligned}
$$

where $p \equiv p_{\mathrm{e}}+p_{\mathrm{i}}$.

Applying $\boldsymbol{B}$. to Eq. (5) yields $\Phi=\Phi(\psi)$. Applying $\boldsymbol{B} \times$ to Eq. (5) yields $\Psi=\Psi(\psi)$ and:

$$
\boldsymbol{v}=\frac{B \Psi^{\prime}}{n} \varepsilon_{\mathrm{F}}+\left(R \Phi^{\prime}+\sqrt{1-\varepsilon_{\mathrm{F}}^{2}} \frac{B \Psi^{\prime}}{n}\right) R \nabla \phi .
$$

Combining Eqs. (8) and (7) yields $\tau=\tau(\psi)$.

Applying $\mu_{0} R^{2} \nabla \phi$. to Eq. (9) yields:

$$
\llbracket I\left|\psi \rrbracket=\llbracket \mu_{0} m_{\mathrm{i}} R v_{\text {tor }} \Psi^{\prime}\right| \psi \rrbracket .
$$

Thus:

$$
I=\left(I_{*}+\mu_{0} m_{\mathrm{i}} R^{2} \Phi^{\prime} \Psi^{\prime}\right) /\left(1-M_{\mathrm{A}, P}^{2}\right),
$$

for some $I_{*}(\psi)$, where $M_{\mathrm{A}, P}$ is the poloidal-Alfvénic Mach number and $V_{\mathrm{A}, P}$ is the poloidal Alfvén velocity:

$$
\begin{aligned}
& M_{\mathrm{A}, P} \equiv v_{\mathrm{pol}} / V_{\mathrm{A}, P}=\Psi^{\prime} \sqrt{\mu_{0} m_{\mathrm{i}} / n} \\
& V_{\mathrm{A}, P} \equiv \varepsilon_{\mathrm{F}} B / \sqrt{\mu_{0} m_{\mathrm{i}} n} .
\end{aligned}
$$

Observe that $I$ is singular when $v_{\mathrm{pol}}=V_{\mathrm{A}, P}$. This is the poloidal-Alfvénic singularity.

Applying $-n^{-1} \boldsymbol{B} \cdot$ to Eq. (9):

$$
n^{-1} \llbracket p|\psi \rrbracket=\llbracket K| \psi \rrbracket,
$$




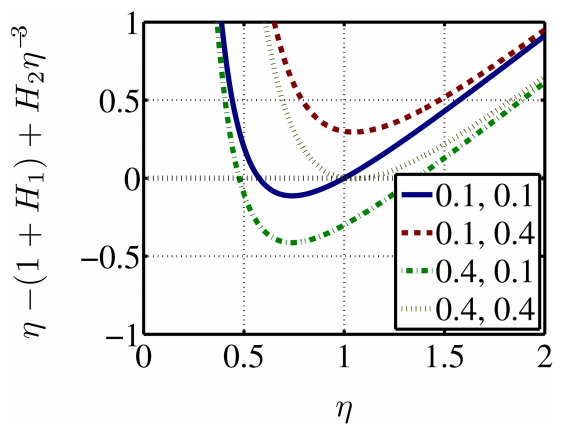

Fig. 1 Sample plots of $\eta-\left(1+H_{1}\right)+H_{2} \eta^{-N}$ vs $\eta$. All of the curves are for $\gamma=5 / 3(N=3)$. The legend entries are the values of $H_{1}$ and $H_{2}$, respectively.

where:

$$
K \equiv \frac{1}{2} m_{\mathrm{i}}\left(R \Phi^{\prime}\right)^{2}-\frac{1}{2} m_{\mathrm{i}}\left(B \Psi^{\prime} / n\right)^{2} .
$$

Observe:

$$
\begin{aligned}
& n^{-1} \llbracket p\left|\psi \rrbracket=n^{-1} \tau \llbracket n^{\gamma}\right| \psi \rrbracket \\
& \quad=[\gamma /(\gamma-1)] \tau \llbracket n^{\gamma-1}|\psi \rrbracket=\llbracket \gamma /(\gamma-1) T| \psi \rrbracket .
\end{aligned}
$$

Thus:

$$
T=T_{*}+[(\gamma-1) / \gamma] K,
$$

for some $T_{*}(\psi)$.

As the physical meaning of $\tau$ is not clear, we rewrite $\tau$ in terms of $n$. Using Eqs. (21) and (30):

$$
n^{\gamma-1}=n_{*}^{\gamma-1}+[\gamma /(\gamma-1)]\left(n_{*} / T_{*}\right) K,
$$

where:

$$
n_{*}=n_{*}(\psi) \equiv\left(T_{*} / \tau\right)^{1 /(\gamma-1)} .
$$

Eq. (31) is the analog of the Bernoulli equation for singlefluid MHD equilibria. However, it cannot be used to directly evaluate $n$ because $K$ depends on $n$ (Eq. (28)). Define $N, H_{1}, H_{2}$ and $\eta$ :

$$
\begin{aligned}
& N \equiv 2 /(\gamma-1), \\
& H_{1} \equiv\left[m_{\mathrm{i}} /\left(N \gamma T_{*}\right)\right]\left(R \Phi^{\prime}\right)^{2}, \\
& H_{2} \equiv\left[m_{\mathrm{i}} /\left(N \gamma T_{*}\right)\right]\left(B \Psi^{\prime} / n_{*}\right)^{2}, \\
& \eta \equiv\left(n / n_{*}\right)^{2 / N} .
\end{aligned}
$$

Note that $H_{2}$ depends on $n$ via $B$ via $I$ (Eq. (24)). However, as long as $v_{\text {pol }} \ll V_{\mathrm{A}, P}$, the dependence will be very weak. With these definitions and a bit of rearranging, Eq. (31) becomes:

$$
\eta-\left(1+H_{1}\right)+H_{2} \eta^{-N}=0 .
$$

The function on the left-hand side of Eq. (37) is illustrated in Fig. 1 for several values of $H_{1}$ and $H_{2}$.

Depending on $H_{1}$ and $H_{2}$, Eq. (37) may have zero, one, or two real roots. Using $\mathrm{d} H_{2} / \mathrm{d} n=0$, the criteria

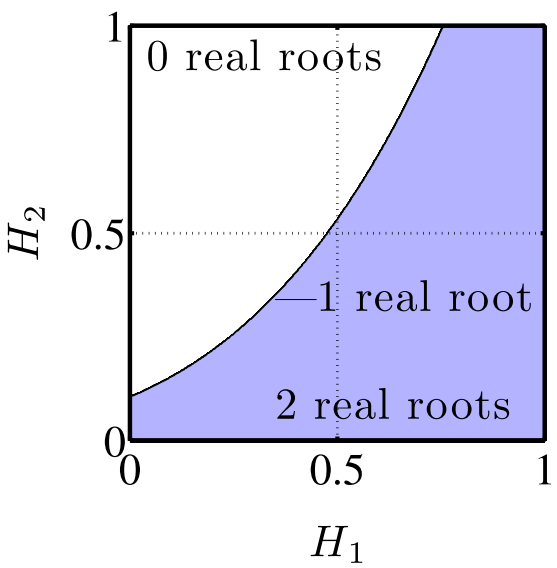

Fig. 2 Contours of the number of real roots to Eq. (37) in $\left(H_{1}, H_{2}\right)$-space for $\gamma=5 / 3(N=3)$. There are no real roots in the white region and there are two real roots in the shaded region. There is one real root on the boundary between the regions.

for the equation to have at least one real root can be found by taking $\mathrm{d} / \mathrm{d} \eta$ :

$$
H_{2} /\left(1+H_{1}\right)^{N+1} \leq N^{N} /(N+1)^{N+1} .
$$

This criteria is illustrated in Fig. 2.

Finally, applying $-\mu_{0} R^{2}\left(\|\nabla \psi\|^{2}\right) \nabla \psi \cdot$ to Eq. (9):

$$
\begin{aligned}
& \left(1-M_{\mathrm{A}, P}^{2}\right) \Delta^{*} \psi+\frac{1}{2}\left(I^{2}\right)^{\prime}+\mu_{0} R^{2} p^{\prime}= \\
& \|\nabla \psi\|^{2}\left(v / V_{\mathrm{A}, P}\right)^{2}(\log R)^{\prime}-\frac{1}{2} M_{\mathrm{A}, P}^{2}\left(\|\nabla \psi\|^{2}\right)^{\prime} .
\end{aligned}
$$

This is the generalized GS equation for single-fluid MHD equilibria with flow.

\section{Relation Between Bernoulli Equa- tion and PS Singularity}

In Sec. 3, we derived a formulation for single-fluid MHD equilibria with flow without using an expansion in $\varepsilon$. In particular, we found that the density must satisfy the Bernoulli equation (Eq. (37)). In this section, we show how the Bernoulli equation is related to the PS singularity in the Ito formulation.

Assume $\varepsilon \ll 1, B_{\text {pol }} \sim \varepsilon B$, and $v_{\text {tor }} \lesssim v_{\text {pol }} \ll V_{\mathrm{A}, P}$. Note that these assumptions are a subset of the ordering assumed for the Ito formulation. From Eq. (22):

$$
\left|R \Phi^{\prime}+\sqrt{1-\varepsilon_{\mathrm{F}}^{2}} B \Psi^{\prime} / n\right| \lesssim \varepsilon_{\mathrm{F}}\left|B \Psi^{\prime} / n\right| .
$$

To the lowest order in $\varepsilon$ [8]:

$$
R \Phi^{\prime}=-B \Psi^{\prime} / n
$$

From the definition of $H_{1}, H_{2}$, and $\eta$ (Eqs. (34)-(36)):

$$
H_{2}=\left(n / n_{*}\right)^{2} H_{1}=\eta^{N} H_{1} \text {. }
$$




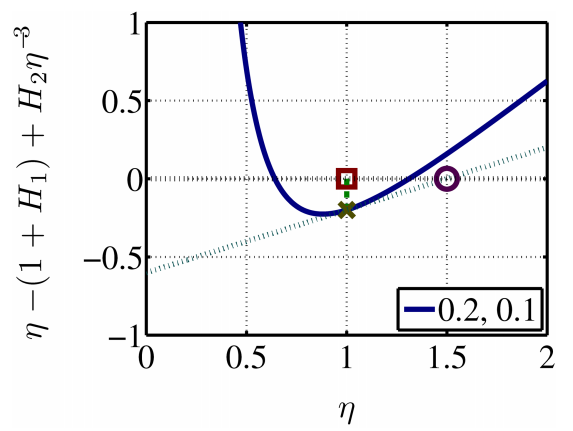

Fig. 3 Graphical illustration of how $\eta_{1}$ is determined for the case $\gamma=5 / 3(N=3), H_{1}=0.2, H_{2}=0.1$. The square is $\eta_{0}$. The circle is $\eta_{0}+\eta_{1}$

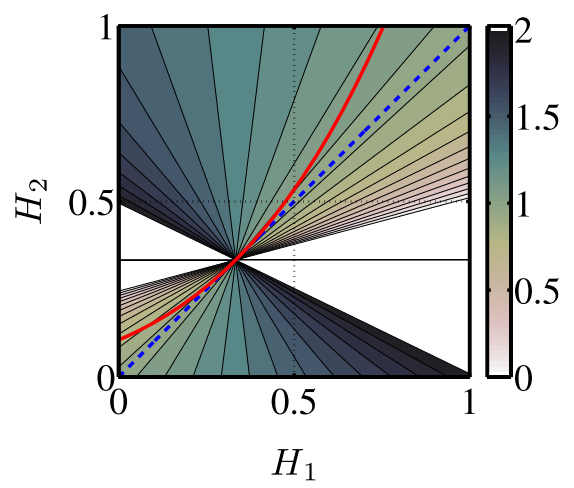

Fig. 4 Contour plot of $\left[(\eta)_{0}+(\eta)_{1}\right]$ in $\left(H_{1}, H_{2}\right)$-space for $\gamma=$ $5 / 3(N=3)$. Contours are shown for $0<\left[(\eta)_{0}+(\eta)_{1}\right]<$ 2 ; contours outside of these bounds have been suppressed. The black line in the suppressed region indicates the singularity in the linear approximation. The dashed blue line is $H_{1}=H_{2}$. The solid red line is the criteria for there to be exactly one real root to the Bernoulli equation.

Eq. (37) yields $\eta=1$ and $H_{1}=H_{2}$.

Consider the next order in $\varepsilon: \eta=(\eta)_{0}+(\eta)_{1}+\ldots$, etc. Under the inverse aspect-ratio expansion, we assume $\left|(\eta)_{1}\right| \ll\left|(\eta)_{0}\right|$, etc. The linearization of Eq. (37) yields:

$$
(\eta)_{1}=\left[\left(H_{1}\right)_{1}-\left(H_{2}\right)_{1}\right] /\left[1-N\left(H_{2}\right)_{0}\right] .
$$

(See Fig. 3 for a graphical representation of the linearization.) Observe that $(\eta)_{1}$ is singular when $N\left(H_{2}\right)_{0}=1$, or, equivalently, when $\left(v_{\mathrm{pol}} / C_{\mathrm{s}, P}\right)_{0}=1$, where $C_{\mathrm{s}, P}$ is the poloidal sound speed (Eq. (10)). This reproduces the poloidal-sonic singularity in the Ito formulation for the single-fluid MHD model.

Comparing the above result with Eq. (37), we see that the singularity in the Ito formulation occurs when there is only one root to the Bernoulli equation (See Fig. 4). This can also be seen in terms of Figs. 1 and 3, when the function is flat at the point $\eta=1$.

Note that, unlike the poloidal-Alfvénic singularity, the poloidal-sonic singularity appears only when using an expansion in $\varepsilon$. The PS singularity results from an inverse aspect-ratio expansion of the Bernoulli equation.

\section{Conclusion}

We have found that, in the single-fluid MHD model, the PS singularity emerges when applying an inverse aspect-ratio expansion to the Bernoulli equation with the constraint $v_{\text {tor }} \lesssim v_{\text {pol }} \ll V_{\mathrm{A}, p}$, and it occurs where the Bernoulli equation has exactly one root. It should be noted that results obtained for the single-fluid MHD model may not necessarily generalize when FLR and two-fluid effects are included. Nevertheless, our analysis suggests the following: in order to calculate MHD equilibria with poloidal-transonic flows, FLR effects, and two-fluid Hall effects, at a minimum, it would be necessary to modify the Ito formulation near the singularity to handle the Bernoulli equation non-linearly.

\section{Acknowledgement}

This work was supported by Grand-in-Aid for Scientific Research (S) (20226017) from JSPS, Japan.

[1] T.S. Taylor, H.S.T. John, A.D. Turnbull et al., Plasma Phys. Control. Fusion 36, B229 (1994).

[2] R. Betti and J.P. Freidberg, Phys. Plasmas 7(6), 2439 (2000).

[3] L. Guazzotto, R. Betti, J. Manickam and S. Kaye, Phys. Plasmas 11(2), 604 (2004).

[4] R. Iacono, A. Bondeson, F. Troyon and R. Gruber, Phys. Fluids B 2, 1794 (1990).

[5] A. Ito and N. Nakajima, AIP Conference Proceedings 1069, 121 (2008).

[6] D. Raburn and A. Fukuyama, Phys. Plasmas 17(2), 122504 (2010).

[7] A. Ito, J.J. Ramos and N. Nakajima, Plasma Fusion Res. 3, 034 (2008).

[8] A. Ito and N. Nakajima, Plasma Phy. Control. Fusion 51, 035007 (2009).

[9] E. Hameiri, Phys. Fluids 26(1), 230 (1983).

[10] J.J. Ramos, Phys. Plasmas 12, 112301 (2005). 